Produto \& Produção, vol. 13 n. 1, p. 148-165 fev. 2012

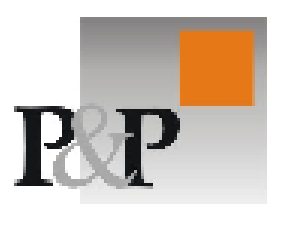

\title{
Estudo das práticas de gerenciamento de projetos voltadas para desenvolvimento de produtos inovadores
}

Recebido em 30/11/2011. Aceito em 20/02/2012.

\section{Samuel Eder}

Departamento de Engenharia de Produção - Escola de Engenharia de São Carlos samuel.eder@oi.com.br

\section{Edivandro Carlos Conforto}

Departamento de Engenharia de Produção - Escola de Engenharia de São Carlos econforto@gmail.com

\section{Juliana Pereira Schnetzler}

Departamento de Engenharia de Produção - Escola de Engenharia de São Carlos juliana9151@gmail.com

\section{Daniel Capaldo Amaral}

Departamento de Engenharia de Produção - Escola de Engenharia de São Carlos amaral@sc.usp.br

\section{Sergio Luis da Silva}

Universidade Federal de São Carlos - UFSCar

Depto. de Ciências da Informação e Programa de Pós-Graduação em Engenharia de Produção

sergiol@ufscar.br

A teoria de gerenciamento de projetos (GP) tem sido alvo de críticas nos últimos anos. Como resultado surgiu novas abordagens, como o gerenciamento ágil de projetos (GAP), acompanhadas de novas práticas, técnicas e ferramentas. Parte delas presente na teoria tradicional de GP e por vezes rebatizada com novos rótulos. A confusão de nomes e práticas diminui a qualidade das pesquisas na área, e estudos nessa temática não conseguem caracterizar precisamente a abordagem utilizada. Este artigo contribui para a solução do problema apresentando uma compilação das práticas, técnicas e ferramentas para planejamento de escopo, extraídas de ambas as teorias, ágil e tradicional. Utilizou-se a revisão bibliográfica sistemática para definição e compilação dos termos práticas, técnicas e ferramentas de GP. O resultado é um referencial do tipo inventário, possibilitando a identificação das práticas em empresas reais e sua categorização quanto à abordagem utilizada, para obterem-se avaliações precisas da contribuição dessa nova abordagem.

Palavras chave: Gerenciamento de Projetos; Gerenciamento Ágil de Projetos; 
Práticas.

The theory of project management (PM) has been target of criticism in recent years. As a result new approaches emerged, how agile project management (APM) followed by new practices, techniques and tools. Some of them present in the traditional theory PM and sometimes renamed with new labels. The confusion of names and practices decreases the quality of research in the area, and studies on this subject cannot precisely characterize the approach used. This paper contributes to the solution of the problem by providing a compilation of practices, techniques and tools for planning scope, extracted from both theories, agile and traditional. We used a systematic literature review to define the terms and compilation practices, techniques and tools for GP. The result is a type inventory reference, enabling the identification practices in real companies and their categorization on the approach used, to obtain accurate assessments of the contribution of this new approach.

Keywords: Project Management; Agile Project Management; Practices.

\section{INTRODUÇÃO}

A teoria de gerenciamento de projetos (GP), juntamente com as primeiras práticas, técnicas e ferramentas surgiram a partir de 1950. Inicialmente aplicadas em grandes projetos, como caso de construção civil, defesa e aeroespacial. Com o passar dos anos a teoria de GP evoluiu (KIOPPENBORG; OPFER, 2002; KOLLTVEIT; KARLSEN; GRONHAUG, 2007; SHENHAR; DVIR, 2007), com o surgimento e consolidação de associações de profissionais da área, cita-se, por exemplo: PMI - Project Management Institute (USA); AIPM - Australian Institute for Project Management (Austrália); IPMA - International Project Management Association (Holanda); APM - Association for Project Management (Reino Unido).

Em geral esses Institutos e Associações atuam na padronização e disseminação dos conhecimentos e práticas de gestão de projetos por meio de "guias de conhecimento". Esses guias, conhecidos como "BoKs - Body of Knowledge" apresentam um conjunto de práticas, técnicas e ferramentas, resumidas em textos normativos, que servem como padrão de terminologia e aplicação, que podem ser utilizadas na maioria dos projetos, sendo rotulados pelos teóricos como a teoria tradicional de gestão de projetos. O mais difundido é o guia PMBOK (Project Management Body of Knowledge) que está em sua quarta edição (PMBOK, 2008).

Nos últimos anos, porém, a literatura de GP tem sido alvo de críticas quanto ao uso generalizado de práticas de GP consideradas tradicionais. Essas práticas apresentariam limitações quando utilizadas em ambientes dinâmicos de negócio, com altos níveis de incertezas e mudanças constantes, justamente onde estão inseridos projetos de produtos inovadores, nos quais raramente o projeto é executado conforme o planejamento inicial (DAWSON; DAWSON, 1998; WILLIAMS, 1999; PERMINOVA; GUSTAFSSON; WIKSTRÖM, 2008; STEFFENS; MARTINSUO; ARTTO, 2007). Nesse contexto, surgiram novas propostas de práticas e técnicas de gestão de projetos utilizando ferramentas visuais, interativas e simplificadas (CHIN, 2004; HIGHSMITH, 2004; AUGUSTINE, 2005; COHN, 2005). Essas práticas, 
técnicas e ferramentas são conhecidas como "metodologias ágeis", pertencentes ao movimento intitulado de "Gerenciamento Ágil de Projetos - GAP", que vem ganhando destaque desde 2001, a partir da assinatura do manifesto para desenvolvimento ágil de software (BECK et al, 2001). O manifesto apresenta um conjunto de princípios e valores que visam melhores condições para se gerenciar projetos em ambientes dinâmicos, com inúmeras incertezas, sujeito a ocorrência de mudanças.

O manifesto é considerado o marco para a disseminação dos métodos ágeis, e inúmeros estudos surgiram após sua criação: Boehm (2002), Griffiths (2005), Hodgetts (2005), Nerur, Mahapatra e Mangalar (2005), Boehm e Turner (2004, 2005), Angioni (2006) e Salo e Abrahamssom (2007). Em sua maioria são semelhantes e descrevem princípios e práticas para o gerenciamento ágil, porém com enfoque exclusivo na área de desenvolvimento de software. Apesar de casos de aplicação na área de software, essa teoria pode ser útil para desenvolvimento de produtos manufaturados, conforme Smith (2007) argumenta em seu livro. Outras evidências dos benefícios da aplicação dessas práticas em projetos de novos produtos inseridos em ambientes de inovação são demonstradas em pesquisas recentes sobre o tema (CONFORTO; AMARAL, 2009; 2010; AMARAL et al (2011).

Este artigo apresenta um levantamento das principais práticas, técnicas e ferramentas para planejamento de escopo, segundo a teoria tradicional e ágil. Ressalta-se que a principal contribuição não está apenas na identificação das práticas, mas na sua classificação quanto à origem. Para o PMBOK (2008), processo de planejamento compreende todos os processos necessários para definir o escopo, objetivos e ações necessários para atingir os resultados do projeto. Por "gerenciamento de escopo" incluem-se os processos necessários para assegurar que o projeto abrange todo o trabalho necessário, e apenas o necessário, para terminar 0 projeto com sucesso. Esse gerenciamento está relacionado principalmente com a definição e controle do que está e não está incluso no projeto (coletar os requisitos, definir o escopo, criar EAP, verificar o escopo, controlar o escopo).

\section{GERENCIAMENTO ÁGIL DE PROJETOS DE PRODUTOS INOVADORES: UMA REVISÃO}

Gerenciar projetos complexos e inovadores por meio da adoção de práticas e ferramentas tradicionais de gerenciamento de projetos tem sido alvo de questionamentos (SUIKKI; TROMSTEDT; HAAPASALO, 2006; DAWSON; DAWSON, 1998; MAYLOR, 2001; ANDERSEN, 1996; WILLIANS, 1999; MAYLOR, 2001; DVIR; RAZ; SHENHAR, 2003; DVIR; LECHLER, 2004; ROZENES; VITNER; SPRAGGETT, 2006; COHN, 2005; SHENHAR; DVIR, 2007). Nota-se que para esses autores as práticas, técnicas e ferramentas ditas tradicionais não são adequadas para esse contexto.

Essas críticas resultaram no surgimento de novas propostas, como por exemplo: "Flexible" (THOMKE; REINERSTEN, 1998; SMITH, 2007), "Adaptive" (SHENHAR; DVIR, 2007), "Iterative" e "Extreme" (DECARLO, 2004; WYSOCKY, 2007), "Lean" (LEACH, 2005), "Agile" (CHIN, 2004; HIGHSMITH, 2004), "Scrum" (SCHWABER, 2004). De maneira geral essas propostas trazem uma simplificação 
das práticas de gerenciamento, o uso de ferramentas visuais e interativas, utilização de poucos padrões, desenvolvimento iterativo com entregas de curto prazo, eliminação de atividades que não agregam valor ao produto/cliente, focando na participação e desenvolvimento das pessoas.

O desafio para explorar essa abordagem no desenvolvimento de produtos manufaturados é identificar as práticas, técnicas e ferramentas existentes, comparálas com a teoria tradicional e avaliar os resultados de aplicação em casos reais. Conforme detalhado em Conforto (2009), é necessário primeiro compreender melhor quais práticas existem, suas diferenças frente aquelas utilizadas na teoria tradicional, para depois conseguir mensurar os resultados.

Contudo, analisando os estudos Mafakheri, Nasiri e Mousavi (2008), Chin (2004) e Ganguly, Nilchiani e Farr (2009) que avaliaram o desempenho do GAP, nota-se que não há um rigor na identificação do nível de uso de tal abordagem pelas empresas. E mais recentemente, Sauser, Reilly e Shenhar (2009), reforçaram esta percepção dizendo que não é possível avaliar mais precisamente a contribuição destas teorias, sem categorizar os diferentes tipos de projeto e relacionar com os diferentes tipos de prática. Falta, portanto, um instrumental teórico que permita a avaliação de qual abordagem de gerenciamento está sendo empregada dentro de uma organização. Nesse sentido, este artigo contribui com este primeiro passo, reunindo as práticas, técnicas e ferramentas existentes na literatura, indicando sua classificação segundo a teoria tradicional ou ágil.

Nesse trabalho adotamos a seguinte definição para o termo "gerenciamento ágil de projetos", voltada para a aplicação em projetos de produtos inovadores (AMARAL et al, 2011): "O gerenciamento ágil de projetos é uma abordagem fundamentada em um conjunto de princípios, cujo objetivo é tornar o processo de gerenciamento de projetos mais simples, flexível e iterativo, de forma a obter melhores resultados em desempenho (tempo, custo e qualidade), menos esforço em gerenciamento e maiores níveis de inovação e agregação de valor para o cliente".

\section{MÉTODO}

O método de pesquisa adotado foi revisão bibliográfica sistemática (RBS), dividido em 3 etapas, proposto por Conforto et al (2011). Na ETAPA 1 foi realizado um levantamento preliminar das fontes de práticas, técnicas e ferramentas indicadas para o gerenciamento de projetos segundo a teoria ágil e tradicional. Foi consultado o trabalho de Conforto e Amaral $(2008,2009)$ como base para a identificação de fontes primárias tais como (PMBOK, 2008; HIGHSMITH; SCHWABER, 2004; MURCH, 2001; COHN, 2005; SMITH, 2007; WYSOCKI, 2007) entre outros. Assim houve uma primeira familiarização com o tema, reunindo um conjunto de práticas iniciais e identificando termos divergentes entre os autores (por exemplo, alguns autores citam "técnica" e outros "método", embora com o mesmo sentido).

$\mathrm{Na}$ segunda (ETAPA 2), foi elaborada uma lista preliminar de periódicos com um total de 87 periódicos. Desse total, 35 periódicos não apresentaram resultado relevante, ou seja, nenhum artigo foi encontrado que apresentasse o uso ou aplicação de uma prática, técnica ou ferramenta de gerenciamento de projetos. A pesquisa foi realizada de 2000 até 2011. Isso porque o manifesto para gerenciamento ágil de projetos foi oficialmente publicado nessa data (BECK et al, 
2001). Então para a presente pesquisa foram considerados 51 periódicos, sendo as buscas realizadas em cada periódico separadamente. Embora somente 27 periódicos apresentaram resultados relevantes para a pesquisa, reuniu-se um total de 8159 artigos que tiveram o título e palavras-chave revisados. Desse conjunto de artigos restaram 141 dos quais 59 foram selecionados para leitura completa.

$\mathrm{Na}$ ETAPA 3 foi realizada uma compilação das práticas, técnicas e ferramentas encontradas durante a revisão bibliográfica sistemática. Os termos "prática", "técnica" e "ferramenta" foram definidos e em seguida foi realizada a leitura completa dos 59 artigos encontrados na revisão. Alem disso, foram consideradas fontes primárias do estudo como base para compilação das práticas, técnicas e ferramentas de gerenciamento de projetos.

\section{RESULTADOS}

Esta seção apresenta os resultados desse estudo organizados da seguinte forma: primeiro são apresentadas as práticas de gerenciamento de projetos; em segundo, as técnicas e por fim as ferramentas.

\subsection{Práticas, técnicas e ferramentas de gerenciamento de projetos}

Como o objetivo deste trabalho é identificar as práticas para gerenciamento de projetos, não somente apresentadas pelo PMBOK (literatura tradicional), mas também outras fontes, foi preciso adotar uma definição para o termo prática. Inicialmente foi consultado o guia PMBOK (2008) de onde foram extraídas as definições básicas. Alguns outros glossários, manuais e livros de renome, como 0 CMMI (Capability Maturity Model Integration) também foram consultados, porém a grande maioria não apresentou uma definição clara do termo que fosse útil nesse estudo. Uma pesquisa em dicionários e bases de palavras da língua inglesa também foi realizada, visando melhorar a compreensão do termo (WORDNET®, PRINCETON UNIVERSITY; OXFORD DICTIONARIES, OXFORD UNIVERSITY PRESS, 2010; THE MERRIAM - WEBSTER DICTIONARY).

O resultado dessa busca e análise do termo "prática" indica duas vertentes. A primeira expressa sua correlação com a frequência de execução de uma determinada atividade. Por exemplo, há especialização e proficiência. A segunda vertente, pautada na descrição do PMBOK (2008) e Huttenlocher e Spoonhower (2002) e pelo dicionário de Cambridge, define o termo como sendo uma "ação ou forma de trabalho", sem fazer correlação com o grau de especialização. Esta segunda vertente foi considerada por se tratar da definição mais adaptada ao contexto de gestão de projetos e mais atrelada ao objetivo deste trabalho.

Portanto, a definição adotada nesse estudo foi uma adaptação da proposta pelo PMBOK (2008): "Prática é um tipo específico de ação profissional ou de gerenciamento que contribui para a execução de um processo e que pode empregar uma ou mais técnicas e ferramentas". Alterou-se o termo para retirar o efeito circular do texto proposto no guia, que inicialmente define-o como um tipo de atividade que 
contribui para a execução de um processo, termo que a própria referência define como sendo um conjunto de atividades.

$\mathrm{Na}$ definição do termo "prática" existe dois elementos chave. O primeiro é "técnica" e o segundo "ferramenta". Para a definição de ambos os termos o mesmo processo utilizado para o termo "prática" foi adotado. Com relação ao termo "técnica" também ficou evidente duas vertentes. A primeira descrevendo-o como "habilidade, pericia, precisão na execução de uma atividade". Na segunda, considera-o como sendo um "método ou maneira de se realizar uma tarefa específica". Por isso, nesse estudo adota-se a definição para o termo "técnica" como: "Técnica é um procedimento sistemático definido usado por um recurso humano para realizar uma atividade a fim de produzir um produto ou resultado ou oferecer um serviço, e que pode empregar uma ou mais ferramentas" (PMBOK, 2008).

Com relação ao termo "ferramentas" não houve diferença nas definições encontradas, optando-se por adotar a proposta pelo PMBOK (2008) por ser a mais difundida e preconizada na teoria de gerenciamento de projetos. Conforme descrito no guia, uma ferramenta é "alguma coisa tangível, como um modelo ou um programa de software, usada na realização de uma atividade para produzir um produto ou resultado". Nesse sentido, ferramenta é algo tangível, podendo assumir também a forma de um software, ou um quadro branco, um caderno, etc.

\subsection{Inventário de práticas, técnicas e ferramentas}

Nesse estudo, a lista de práticas, técnicas e ferramentas é intitulada de "inventário". Foram criados três inventários, um para cada item. Os inventários são conjuntos união, contendo práticas, técnicas e ferramentas de ambas as abordagens, tradicional e ágil, proveniente de um estudo sistemático de livros e artigos. Os inventários foram construídos com base em 14 fontes distintas, seguindo a classificação proposta pelos autores, tendo como objetivo buscar e compilar as práticas. A descrição de cada item, bem como exemplos de aplicação poderão ser temas de trabalhos futuros nessa área. É importante ressaltar que os demais trabalhos levantados durante a RBS estão sendo analisados.

Apresenta-se, inicialmente, o inventário de práticas, seguido do inventário de técnicas e de ferramentas. Em muitos textos os autores não mencionam quais técnicas ou ferramentas podem ser utilizadas em conjunto com a prática apresentada. A classificação foi feita pelos pesquisadores, segundo a definição adotada no estudo e apresentada na seção anterior (4.1), a prática compreende o uso de uma ou mais técnicas ou ferramentas, no entanto, esse padrão não é comum na literatura de ambas as teorias.

É importante ressaltar que algumas práticas, técnicas e ferramentas foram mantidas os nomes no idioma inglês para preservar a originalidade e possibilitar a identificação da mesma na fonte original do estudo.

A Tabela 1 mostra no lado esquerdo as práticas de planejamento de escopo encontradas na literatura, segundo a abordagem tradicional de gerenciamento de projetos. No total foram identificadas 11 práticas, denominadas pelo código PT (prática tradicional). É importante enfatizar que algumas práticas possuem mais de uma fonte por serem idênticas, apresentadas com nomes diferentes, sendo agrupadas por apresentarem a mesma sistemática. O mesmo agrupamento ocorre 
em caso de serem apresentadas em idioma e por autores diferentes. O lado direito apresenta o conjunto de práticas para planejamento de escopo segundo a teoria de gerenciamento ágil de projetos. No total foram encontradas 8 práticas, denominadas pelo código PA (prática ágil). É possível identificar práticas que podem ser úteis em projetos de produtos manufaturados, como é o caso das práticas PA1, PA4, PA5 e PA6. Existe também sobreposição de algumas práticas, como é o caso da PA4 e PA7, onde o objetivo final é colaborar para criar uma "visão" do produto do projeto, porém não são idênticas por apresentarem sistemáticas diferentes, conforme descrito pelos próprios autores. É importante ressaltar que tanto as práticas, técnicas e ferramentas apresentadas na mesma linha (PT1 e PA1; TT1 e TA1) e assim sucessivamente não possuem nenhuma equivalência.

Tabela 1 - Práticas pertencentes à literatura de GP classificadas segundo as abordagens tradicional e ágil.

\begin{tabular}{|c|c|c|}
\hline $\begin{array}{c}\text { Cod } \\
.\end{array}$ & $\begin{array}{c}\text { Práticas } \\
\text { (TRADICIONAL) }\end{array}$ & Fontes \\
\hline PT1 & Coletar requisitos & $\begin{array}{c}\text { PMBOK } \\
(2008)\end{array}$ \\
\hline PT2 & Definir Escopo & $\begin{array}{c}\text { PMBOK } \\
(2008) \\
\text { Chicarino } \\
(2005) \\
\end{array}$ \\
\hline PT3 & $\begin{array}{l}\text { Definir as } \\
\text { Atividades }\end{array}$ & $\begin{array}{c}\text { PMBOK } \\
(2008) \\
\text { Chicarino } \\
(2005)\end{array}$ \\
\hline PT4 & $\begin{array}{l}\text { Criar EAP } \\
\text { (Estrutura } \\
\text { analítica do } \\
\text { Projeto / WBS } \\
\text { (Work Breakdown } \\
\text { Structure) }\end{array}$ & $\begin{array}{l}\text { PMBOK } \\
(2008) \\
\text { Wysocki } \\
(2007)\end{array}$ \\
\hline PT5 & $\begin{array}{l}\text { Criando o } \\
\text { "Project Overview } \\
\text { Statement" / } \\
\text { Definir os } \\
\text { objetivos do } \\
\text { projeto }\end{array}$ & $\begin{array}{l}\text { Wysocki } \\
\text { (2007) }\end{array}$ \\
\hline PT6 & $\begin{array}{l}\text { Declarar o } \\
\text { Problema/Oportu } \\
\text { nidade }\end{array}$ & $\begin{array}{c}\text { Wysocki } \\
\text { (2007) }\end{array}$ \\
\hline PT7 & $\begin{array}{l}\text { Identify Business } \\
\text { Objectives and } \\
\text { Information } \\
\text { Strategy }\end{array}$ & $\begin{array}{l}\text { Murch } \\
\text { (2001) }\end{array}$ \\
\hline PT8 & $\begin{array}{l}\text { Develop } \\
\text { Conceptual } \\
\text { Design }\end{array}$ & $\begin{array}{l}\text { Murch } \\
\text { (2001) }\end{array}$ \\
\hline PT9 & Investigate & Murch \\
\hline
\end{tabular}

\begin{tabular}{|c|c|c|}
\hline Cod & Práticas (ÁGIL) & Fontes \\
\hline PA1 & Feature Cards & $\begin{array}{l}\text { Highsmith } \\
(2004)\end{array}$ \\
\hline PA2 & $\begin{array}{l}\text { Release, } \\
\text { Milestone, and } \\
\text { Iteration Plan }\end{array}$ & $\begin{array}{l}\text { Highsmith } \\
(2004)\end{array}$ \\
\hline PA3 & $\begin{array}{l}\text { Story Points/ldeal } \\
\text { days }\end{array}$ & Cohn (2005) \\
\hline PA4 & Criar a Visão & $\begin{array}{l}\text { Schwaber } \\
(2004)\end{array}$ \\
\hline PA5 & $\begin{array}{l}\text { Priorizar Product } \\
\text { Backlog }\end{array}$ & $\begin{array}{l}\text { Schwaber } \\
(2004)\end{array}$ \\
\hline PA6 & $\begin{array}{l}\text { Criar Sprint } \\
\text { Backlog }\end{array}$ & $\begin{array}{l}\text { Schwaber } \\
(2004)\end{array}$ \\
\hline PA7 & Lagomizing & $\begin{array}{c}\text { Berggren et } \\
\text { al (2008) }\end{array}$ \\
\hline PA8 & $\begin{array}{l}\text { Organic } \\
\text { integration }\end{array}$ & $\begin{array}{c}\text { Berggren et } \\
\text { al (2008) }\end{array}$ \\
\hline
\end{tabular}




\begin{tabular}{|c|l|c|}
\hline & $\begin{array}{l}\text { Packaged } \\
\text { Systems } \\
\text { Alternatives }\end{array}$ & (2001) \\
\hline PT1 & $\begin{array}{l}\text { Evaluate } \\
\text { Development } \\
0\end{array}$ & $\begin{array}{l}\text { Murch } \\
\text { Alternatives }\end{array}$ \\
\hline PT1 & Finalize Project & $\begin{array}{l}\text { Murch } \\
(2001)\end{array}$ \\
\hline 1 & Work Plan & (2001) \\
\hline
\end{tabular}

A Tabela 2 apresenta no lado esquerdo conjunto de técnicas para planejamento de escopo segundo a teoria tradicional de gerenciamento de projetos. No total foram identificadas 24 técnicas, denominadas pelo código TT (técnica tradicional). É importante enfatizar que as técnicas "decomposição" (proposta pelo PMBOK) e "bottom-up estimates" (proposta por Wysocki) foram reunidas em uma única técnica por se tratarem da mesma sistemática segundo os autores, dividir as tarefas em partes menores. O lado direito apresenta o conjunto de técnicas para planejamento de escopo segundo a teoria de gerenciamento ágil de projetos. No total foram identificadas 24 técnicas, denominadas pelo código TA (técnica ágil). Ressalta-se que as técnicas "reunião" (proposta por Smith/Cohn) e "dayly scrum" (proposta por Schwaber) não foram agrupadas por se tratarem de sistemáticas diferentes segundo os autores, embora ambas tratem de reuniões, a técnica proposta por Schwaber é prescrita por padrões de perguntas, algo não mencionado por Smith/Cohn. Assim como nas práticas da literatura ágil, também é possível identificar técnicas que possam ser úteis em projetos de produtos manufaturados, sendo as TA2, TA3, TA4, TA5, TA10, TA20 e TA23

Tabela 2 - Técnicas pertencentes à literatura de GP classificadas segundo as abordagens tradicional e ágil.

\begin{tabular}{|c|c|c|c|c|c|}
\hline Cod. & $\begin{array}{c}\text { Técnicas } \\
\text { (TRADICIONAL) }\end{array}$ & Fontes & Cod. & Técnicas (ÁGIL) & Fontes \\
\hline TT1 & Entrevistas & $\begin{array}{l}\text { PMBOK } \\
(2008)\end{array}$ & TA1 & $\begin{array}{l}\text { Opinião } \\
\text { especializada }\end{array}$ & $\begin{array}{l}\text { Cohn } \\
\text { (2005) }\end{array}$ \\
\hline TT2 & $\begin{array}{l}\text { Dinâmicas de } \\
\text { grupo }\end{array}$ & $\begin{array}{c}\text { PMBOK } \\
(2008)\end{array}$ & TA2 & "Planning Poker" & $\begin{array}{l}\text { Cohn } \\
(2005)\end{array}$ \\
\hline TT3 & $\begin{array}{l}\text { Opinião } \\
\text { Especializada / } \\
\text { Oficina }\end{array}$ & $\begin{array}{l}\text { PMBOK } \\
(2008)\end{array}$ & TA3 & $\begin{array}{l}\text { Estimar por } \\
\text { comparação }\end{array}$ & $\begin{array}{l}\text { Cohn } \\
\text { (2005) }\end{array}$ \\
\hline TT4 & $\begin{array}{l}\text { Técnicas de } \\
\text { criatividade em } \\
\text { grupo }\end{array}$ & $\begin{array}{l}\text { PMBOK } \\
(2008)\end{array}$ & TA4 & Run an Iteration & $\begin{array}{l}\text { Cohn } \\
\text { (2005) }\end{array}$ \\
\hline TT5 & $\begin{array}{l}\text { Técnicas de } \\
\text { tomada de } \\
\text { decisão em } \\
\text { grupo }\end{array}$ & $\begin{array}{l}\text { PMBOK } \\
(2008)\end{array}$ & TA5 & Make a Forecast & $\begin{array}{l}\text { Cohn } \\
\text { (2005) }\end{array}$ \\
\hline TT6 & $\begin{array}{l}\text { Estimar por } \\
\text { analogia }\end{array}$ & $\begin{array}{c}\text { PMBOK } \\
(2008)\end{array}$ & TA6 & $\begin{array}{l}\text { Estimar por } \\
\text { desagregação }\end{array}$ & $\begin{array}{l}\text { Cohn } \\
(2005)\end{array}$ \\
\hline
\end{tabular}




\begin{tabular}{|c|c|c|c|c|c|}
\hline TT7 & $\begin{array}{l}\text { Modelo (Lista de } \\
\text { Atividades) }\end{array}$ & $\begin{array}{c}\text { PMBOK } \\
(2008)\end{array}$ & TA7 & $\begin{array}{l}\text { Identificar os } \\
\text { trabalhos que } \\
\text { podem ser } \\
\text { finalizados }\end{array}$ & $\begin{array}{l}\text { Cohn } \\
(2005)\end{array}$ \\
\hline TT8 & Business case & $\begin{array}{c}\text { PMBOK } \\
(2008)\end{array}$ & TA8 & $\begin{array}{l}\text { Identificar as } \\
\text { condições de } \\
\text { satisfação }\end{array}$ & $\begin{array}{l}\text { Cohn } \\
(2005)\end{array}$ \\
\hline TT9 & $\begin{array}{l}\text { Análise de } \\
\text { produto }\end{array}$ & $\begin{array}{c}\text { PMBOK } \\
(2008)\end{array}$ & TA9 & Daily Scrum & $\begin{array}{c}\text { Schwaber } \\
(2004)\end{array}$ \\
\hline TT10 & $\begin{array}{l}\text { Decomposição / } \\
\text { Bottom-up } \\
\text { estimates }\end{array}$ & $\begin{array}{c}\text { PMBOK } \\
(2008) \\
\text { Wysocki } \\
(2007)\end{array}$ & TA10 & Product Backlog & $\begin{array}{c}\text { Schwaber } \\
(2004)\end{array}$ \\
\hline TT11 & $\begin{array}{l}\text { Inspeção / } \\
\text { Observação }\end{array}$ & $\begin{array}{c}\text { PMBOK } \\
(2008)\end{array}$ & TA11 & Reunião & $\begin{array}{l}\text { Smith } \\
(2007) \\
\text { Cohn } \\
(2005) \\
\end{array}$ \\
\hline TT12 & Noun-type & $\begin{array}{l}\text { Wysocki } \\
(2007)\end{array}$ & TA12 & $\begin{array}{l}\text { Separar os } \\
\text { componentes de } \\
\text { alto risco }\end{array}$ & $\begin{array}{l}\text { Hass } \\
(2009)\end{array}$ \\
\hline TT13 & Verb-type & $\begin{array}{l}\text { Wysocki } \\
(2007)\end{array}$ & TA13 & $\begin{array}{l}\text { Progressively } \\
\text { Elaborate the } \\
\text { Plan }\end{array}$ & $\begin{array}{l}\text { Hass } \\
(2009)\end{array}$ \\
\hline TT14 & Organizational & $\begin{array}{c}\text { Wysocki } \\
(2007)\end{array}$ & TA14 & $\begin{array}{l}\text { Estimar por } \\
\text { Intuição }\end{array}$ & $\begin{array}{l}\text { Hass } \\
(2009)\end{array}$ \\
\hline TT15 & \begin{tabular}{|l|} 
Joint Project \\
Planning Session
\end{tabular} & $\begin{array}{l}\text { Wysocki } \\
\text { (2007) }\end{array}$ & TA15 & Entrevistas & $\begin{array}{l}\text { Hass } \\
(2009)\end{array}$ \\
\hline TT16 & Delphi Technique & $\begin{array}{l}\text { Wysocki } \\
\text { (2007) }\end{array}$ & TA16 & Experimento & $\begin{array}{l}\text { Highsmith } \\
\text { (2004) }\end{array}$ \\
\hline TT17 & $\begin{array}{l}\text { Árvores de } \\
\text { decisão }\end{array}$ & $\begin{array}{l}\text { Chicarino } \\
(2005)\end{array}$ & TA17 & \begin{tabular}{|l} 
Product \\
Architecture
\end{tabular} & $\begin{array}{l}\text { Highsmith } \\
\text { (2004) }\end{array}$ \\
\hline TT18 & $\begin{array}{l}\text { Earned Value } \\
\text { Management }\end{array}$ & $\begin{array}{c}\text { Chicarino } \\
(2005) \\
\text { Carden } \\
(2008) \\
\end{array}$ & TA18 & $\begin{array}{l}\text { Product Feature } \\
\text { List }\end{array}$ & $\begin{array}{l}\text { Highsmith } \\
\text { (2004) }\end{array}$ \\
\hline TT19 & $\begin{array}{l}\text { Critical Path } \\
\text { Method }\end{array}$ & $\begin{array}{c}\text { Chicarino } \\
(2005)\end{array}$ & TA19 & $\begin{array}{l}\text { Expandir a } \\
\text { "product } \\
\text { breakdown } \\
\text { structure" }\end{array}$ & $\begin{array}{l}\text { Highsmith } \\
(2004)\end{array}$ \\
\hline TT20 & $E A B$ & $\begin{array}{c}\text { Chicarino } \\
(2005)\end{array}$ & TA20 & Feature cards & $\begin{array}{l}\text { Highsmith } \\
\text { (2004) }\end{array}$ \\
\hline TT21 & $\begin{array}{l}\text { Business } \\
\text { problem definition }\end{array}$ & $\begin{array}{c}\text { Besner et al } \\
(2008)\end{array}$ & TA21 & Simulações & $\begin{array}{l}\text { Highsmith } \\
\text { (2004) }\end{array}$ \\
\hline TT22 & Reunião & $\begin{array}{l}\text { Besner et al } \\
(2008)\end{array}$ & TA22 & $\begin{array}{l}\text { Modelo (Lista de } \\
\text { Atividades) }\end{array}$ & $\begin{array}{l}\text { Highsmith } \\
\text { (2004) }\end{array}$ \\
\hline TT23 & $\begin{array}{l}\text { Método da } \\
\text { cadeia crítica }\end{array}$ & $\begin{array}{c}\text { Besner et al } \\
(2008)\end{array}$ & TA23 & $\begin{array}{l}\text { Product Vision } \\
\text { Box }\end{array}$ & $\begin{array}{l}\text { Highsmith } \\
\text { (2004) }\end{array}$ \\
\hline TT24 & Milestone & Besner et al & \begin{tabular}{|l} 
TA24 \\
\end{tabular} & Project Data & Highsmith \\
\hline
\end{tabular}




\begin{tabular}{|l|l|l|l|}
\hline planning & $(2008)$ \\
\hline
\end{tabular}

Ao se analisar a Tabela 1 (práticas) e Tabela 2 (técnicas) nota-se que Highsmith (2004) propõe o mesmo termo "feature cards", isso acontece pois primeiramente ocorre uma coleta de informações básicas sobre os recursos (prática), após isso as informações dos cartões são identificadas e inseridos dados como: nomes, descrição, tipo, esforço estimado de trabalho, requisitos incertos, dependências e testes de aceitação (técnica).

Outra peculiaridade existe na Tabela 2, onde "reunião" (TT22 e TA11); "modelo - lista de atividades" (TT7 e TA22) e "opinião especializada" (TT3 e TA1) é mencionada como uma técnica utilizada tanto na teoria tradicional quanto na ágil, indicando indícios que algumas técnicas já existiam antes do GAP, e não necessariamente podem ser consideradas ágil. Porém, o que pode diferenciar uma da outra é a ferramenta de apoio, por exemplo, um software, ou o uso de um quadro com recados auto-adesivos, permitindo a interação entre os membros, princípio regido pelo GAP.

Durante o estudo, além das técnicas específicas indicadas pela literatura ágil e tradicional, foram identificadas algumas técnicas mais genéricas sem nenhuma indicação de uso específico e não mencionando nenhuma classificação segundo o autor, nem mesmo a utilização de alguma referência classificada ágil ou tradicional. Foram consideradas, portanto, como úteis tanto em ambientes e abordagens cunhadas na teoria ágil quanto tradicional. Exemplos dessas técnicas são propostas por Carden et al (2008), sendo: Benchmarking; Balanced Scorecard; Critical Cloud Method; Work Packages. Outras são propostas por Sibbet (2010), sendo: Comparing Pairs e Dot Voting.

A Tabela 3 apresenta no lado esquerdo o conjunto de ferramentas para planejamento de escopo segundo a teoria tradicional de GP. No total foram identificadas 11 ferramentas, denominadas pelo código FT (ferramenta tradicional). O lado direito apresenta o conjunto de ferramentas para planejamento de escopo segundo a teoria ágil de GP. No total foram identificadas 11 ferramentas, denominadas pelo código FA (ferramenta ágil). Ressalta-se que a ferramenta "planilha" (proposta por Schwaber /Besner) e "tabelas" (proposta por Cohn/Wysocki) foram agrupadas por se tratarem da mesma sistemática segundo os autores, em geral utilizando-se uma folha, quadro ou formulário para o lançamento de informações. Assim como nas práticas e técnicas da literatura ágil, também é possível identificar ferramentas que possam ser úteis em projetos de produtos manufaturados, sendo as FA2, FA6 e FA8.

Tabela 3 - Ferramentas pertencentes à literatura de GP classificadas segundo as abordagens tradicional e ágil

\begin{tabular}{|c|c|c|c|c|c|}
\hline Cod. & $\begin{array}{c}\text { Ferramentas } \\
\text { (TRADICIONAL) }\end{array}$ & Fontes & Cod. & $\begin{array}{l}\text { Ferramentas } \\
\text { (ÁGIL) }\end{array}$ & Fontes \\
\hline FT1 & $\begin{array}{l}\text { Manual da } \\
\text { qualidade } \\
\text { (manual de } \\
\text { procedimentos) }\end{array}$ & $\begin{array}{l}\text { Besner et } \\
\text { al (2008) }\end{array}$ & FA1 & Planilha/Tabela & $\begin{array}{c}\text { Schwaber } \\
(2004) \\
\text { Cohn }(2005)\end{array}$ \\
\hline FT2 & Diagrama & Besner et & FA2 & Cards Planning & Cohn (2005) \\
\hline
\end{tabular}




\begin{tabular}{|c|c|c|c|c|c|}
\hline & $\begin{array}{l}\text { (Pareto/Causa e } \\
\text { Efeito)/Gráfico }\end{array}$ & al (2008) & & Poker & \\
\hline FT3 & Lista & $\begin{array}{l}\text { Besner et } \\
\text { al (2008) }\end{array}$ & FA3 & Diagrama/Gráfico & $\begin{array}{c}\text { Schwaber } \\
(2004) \\
\text { Cohn (2005) }\end{array}$ \\
\hline FT4 & Contrato & $\begin{array}{l}\text { Besner et } \\
\text { al (2008) }\end{array}$ & FA4 & Lista & Cohn (2005) \\
\hline FT5 & Relatório & $\begin{array}{l}\text { Besner et } \\
\text { al (2008) }\end{array}$ & FA5 & $\begin{array}{l}\text { Cartão/Note } \\
\text { Cards }\end{array}$ & $\begin{array}{c}\text { Highsmith } \\
(2004) \\
\text { Cohn (2005) }\end{array}$ \\
\hline FT6 & Planilha/Tabela & $\begin{array}{c}\text { Besner et } \\
\text { al (2008) } \\
\text { Wysocki } \\
(2007)\end{array}$ & FA6 & Esboço & $\begin{array}{l}\text { Highsmith } \\
(2004)\end{array}$ \\
\hline FT7 & Banco de Dados & $\begin{array}{l}\text { Wysocki } \\
(2007) \\
\text { Besner et } \\
\text { al (2008) }\end{array}$ & FA7 & Questionário & $\begin{array}{l}\text { Highsmith } \\
\text { (2004) }\end{array}$ \\
\hline FT8 & Quadros & $\begin{array}{l}\text { Wysocki } \\
(2007)\end{array}$ & FA8 & Maquetes & $\begin{array}{l}\text { Highsmith } \\
(2004)\end{array}$ \\
\hline FT9 & $\begin{array}{l}\text { Cartão/Note } \\
\text { cards }\end{array}$ & $\begin{array}{l}\text { Wysocki } \\
(2007)\end{array}$ & FA9 & Quadros & $\begin{array}{c}\text { Schwaber } \\
(2004)\end{array}$ \\
\hline FT10 & Questionário & $\begin{array}{c}\text { PMBOK } \\
(2008)\end{array}$ & FA10 & $\begin{array}{l}\text { Feature } \\
\text { Breakdown } \\
\text { Structure }\end{array}$ & $\begin{array}{l}\text { Highsmith } \\
(2004)\end{array}$ \\
\hline FT11 & $\begin{array}{l}\text { Softwares para } \\
\text { gerenciamento } \\
\text { de projetos }\end{array}$ & $\begin{array}{c}\text { Chicarino } \\
(2005)\end{array}$ & FA11 & Cartões & $\begin{array}{l}\text { Highsmith } \\
(2004)\end{array}$ \\
\hline
\end{tabular}

Analisando-se a Tabela 3, notam-se várias ferramentas mencionadas tanto na teoria tradicional quanto na ágil, como no caso da ferramenta (FT2 e FA3), (FT3 e FA4), (FT6 e FA1), (FT8 e FA9) e por último (FT9 e FA5).

Novamente as análises apontam indícios que algumas ferramentas já existiam antes do GAP, cabendo analisar as diferenças em como essas ferramentas são executadas, se regem os princípios do GAP, para então serem consideradas ágil.

Durante o estudo das ferramentas, também foram encontradas ferramentas genéricas sem nenhum uso específico ou não mencionando nenhuma classificação segundo os autores. Novamente esses autores não utilizaram nenhuma referência classificada ágil ou tradicional, podendo ser úteis tanto em ambientes e abordagens cunhadas na teoria ágil quanto tradicional. Exemplos dessas ferramentas são propostas por Carden et al (2008), sendo: Milestone Chart; Software de processamento de texto, ferramentas de apresentação. Outras são propostas por Sibbet (2010), sendo: Ata de Reunião; Checklist; E-mail; Protótipo/Template; Mental models/Process Maps. Algumas citadas pelo autor como: Radar diagrams; Vintage Charts; Vision Murals apresentam bastante a questão visual, princípio regido no GAP. 
A Figura 1 ilustra dois gráficos que compilam os resultados dos inventários. Ao se analisar o gráfico percebe-se que técnicas e ferramentas possuem as mesmas quantidades difundidas na literatura tradicional de gerenciamento de projetos quanto no GAP, porém isso não acontece nas práticas. Essa explicação se dá pelo fato de vários autores da teoria ágil enfatizarem simplicidade na gestão, como exemplo o termo "visão" ao invés de escopo, sendo que a prática "criar a visão" muitas vezes engloba um conjunto de práticas, algumas delas tradicionais, como no caso de "coletar requisitos" e "definir escopo". Segundo Amaral et al (2011) assim como o escopo a visão tem o papel de descrever quais resultados o projeto precisa atingir, apresentando qualidades adicionais não citadas na teoria tradicional de escopo do projeto.

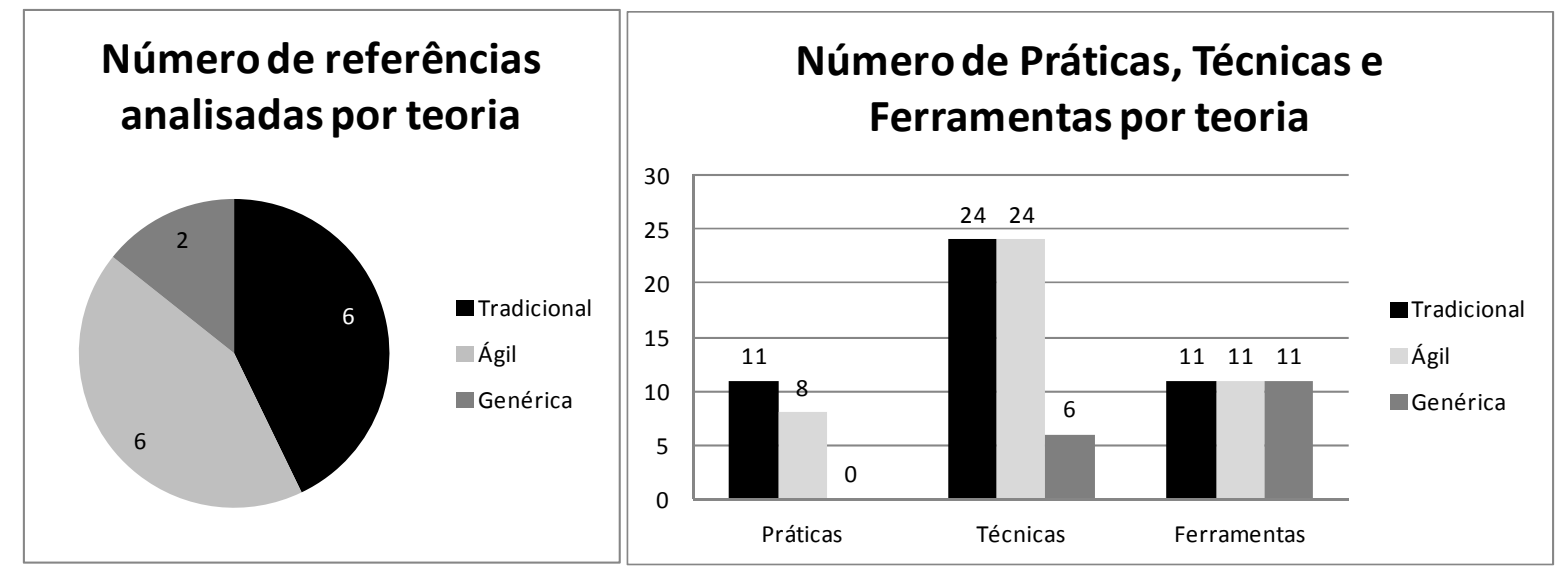

Figura 1 - Demonstração gráfica dos autores analisados e resultados obtidos

A Figura 2 apresenta, conforme literatura estudada, a quantidade de práticas, técnicas e ferramentas semelhantes, que apresentam igualdade por serem a mesma difundida em ambas as teorias (mesmo nome e mesma sistemática), ou que embora possuam nomes diferentes apresentam a mesma sistemática conforme os autores, como por exemplo, FT16 e FA10, que possuem como intuito descrever matérias, componentes e sub componentes. Ressalta que não foram consideradas práticas, técnicas e ferramentas com o mesmo objetivo, por haver determinadas situações em que os objetivos são os mesmos, como em escopo e visão, ambas tem o papel de descrever quais resultados o projeto precisa atingir, porém apresentam sistemáticas completamente divergentes. Vale lembrar que na execução dessas práticas, técnicas e ferramentas em casos reais pode haver diferenças, se rege os princípios do GAP, como iteração entre os membros, podendo então serem consideradas ágil. 


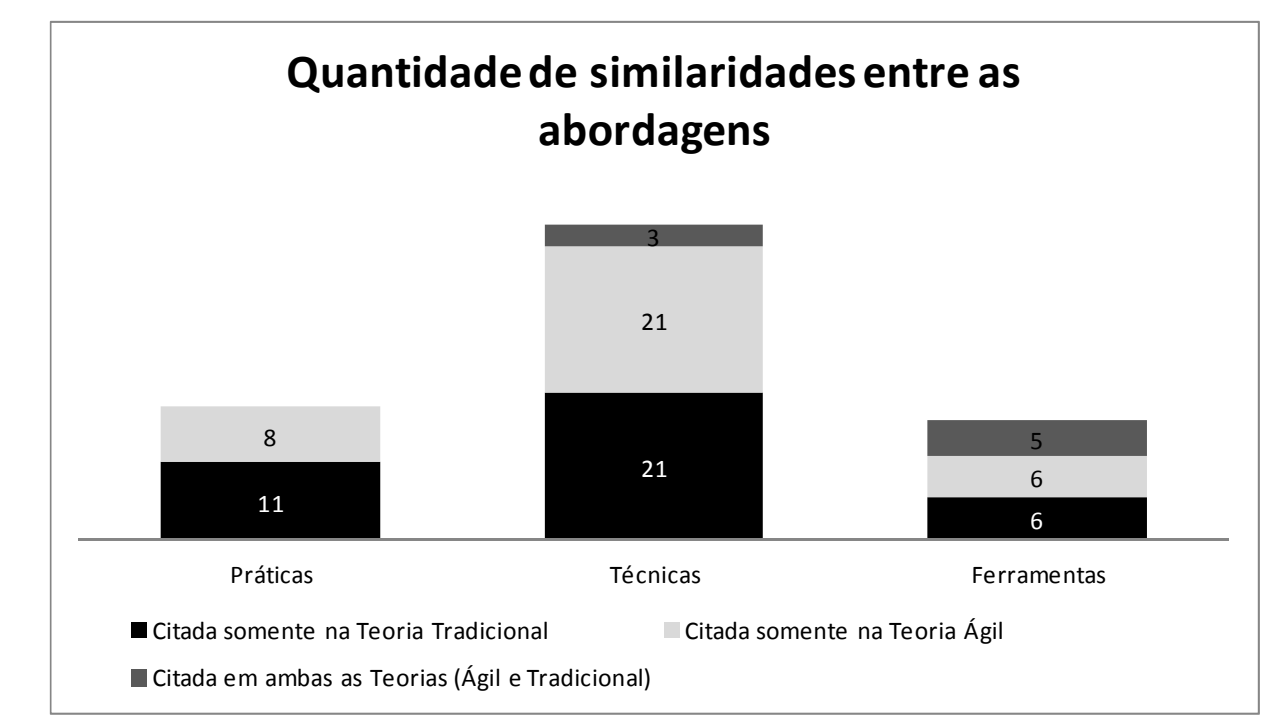

Figura 2 - Quantidade de práticas, técnicas e ferramentas similares por abordagem

Ao analisar o gráfico percebe-se que não existe similaridade nas práticas. No caso das técnicas e ferramentas propostas, existe um número pequeno de similaridades no caso das técnicas, precisamente 3 técnicas idênticas (TT22 e TA11), (TT7 e TA22) e (TT3 e TA1) e 5 ferramentas (FT2 e FA3), (FT3 e FA4), (FT6 e FA1), (FT8 e FA9) e (FT9 e FA5) entre as duas teorias, indicando que realmente a teoria de GAP propõem práticas e técnicas consideradas específicas e mais indicadas para planejamento de projetos inovadores, cabendo a outros estudos avaliar padrões de práticas, técnicas e ferramentas que possam ser úteis no gerenciamento de projetos e como essas são utilizadas pelas empresas no gerenciamento.

\section{CONSIDERAÇÕES FINAIS}

O artigo serve de alerta para os pesquisadores e profissionais interessados no estudo do Gerenciamento Ágil de Projetos. Por meio de revisão bibliográfica sistemática e ampla, demonstra a necessidade de obtenção de meios mais rigorosos de aferir qual a abordagem de GP, práticas, técnicas e ferramentas estão sendo utilizadas pela empresa, antes de se comparar desempenho e a contribuição das abordagens.

Os resultados alcançados demonstram também um caminho para a solução deste problema de pesquisa, verificando que é possível obter-se um inventário de práticas de GP, classificadas segundo as teorias de origem. Um inventário deste tipo poderia ser útil em campo para identificar de maneira mais precisa a forma como uma empresa gerencia os seus projetos, e como são combinadas as práticas, com as técnicas e ferramentas mais adequadas.

Nesta primeira versão foram compiladas 19 práticas, 54 técnicas e 33 ferramentas para planejamento de escopo envolvendo as abordagens ágil, tradicional e estudos que não se enquadraram em nenhuma das abordagens sendo classificados como neutros. 
Os resultados devem ser tomados, entretanto, como preliminares. Em primeiro lugar porque as análises de similaridade deverão continuar e serem aprofundadas. Há limitações quanto à descrição da prática, técnica e ferramenta na literatura de ambas as abordagens, tradicional e ágil. Faz-se necessário uma comparação entre as práticas, técnicas e ferramentas tanto em termos teóricos, analisando-se estudos que utilizaram determinadas práticas para o gerenciamento de projetos inovadores.

O segundo aspecto é que a criação do inventário tal qual proposto depende do seu uso em campo, isto é, a verificação da precisão e praticidade de uso destas práticas na identificação de ações realizadas por empresas reais. Este trabalho está sendo realizado no momento pela equipe de pesquisa, como parte de uma nova etapa desse estudo.

Apesar das ressalvas, o nível de evolução alcançado permite que profissionais de empresa já façam uso desta primeira versão das práticas, para diagnosticar e propor melhorias em suas organizações.

Por fim, há outra perspectiva de trabalho futuro, a realização de esforço similar para as demais áreas do gerenciamento de projetos, como riscos, aquisição, comunicação, etc.

\section{AGRADECIMENTOS}

Os autores agradecem o apoio financeiro da Fundação de Amparo à Pesquisa do Estado de São Paulo (FAPESP), ao Conselho Nacional de Desenvolvimento Científico e Tecnológico (CNPq) e Coordenação de Aperfeiçoamento de Pessoal de Nível Superior (CAPES).

\section{REFERÊNCIAS}

AMARAL, D. C.; CONFORTO, E. C.; BENASSI, J. L. C.; ARAÚJO, C. Gerenciamento Ágil de Projetos - aplicação em produtos inovadores. São Paulo: Saraiva, 2011.

ANDERSEN, E. Warning: activity planning is hazardous to your project's health! International Journal of Project Management, v. 14, n. 2, p. 89-94, 1996.

ANGIONI, M.; CARBONI, D.; PINNA, S.; SANNA, R.; SERRA, N.; SORO, A. Integrating XP project management in development environments. Journal of Systems Architecture, v. 52, n. 11, p. 619-626, 2006.

AUGUSTINE, S. Managing Agile Projects. Virginia: Prentice Hall PTR, 2005.

BECK, K.; BEEDLE, M.; VAN BENNEKUM, A.; et al. Manifesto for agile software development. 2001. Disponível em 
<http://www.xprogramming.com/publications/dc9810cs.pdf> Acesso em 19 jan. 2007.

BESNER, C.; HOBBS, B. Discriminating Contexts and Project Management Best Practices on Innovative and Noninnovative Projects. Project Management Journal, v. 39 , p. 123-134, 2008.

BESNER , C.; HOBBS, B. The Perceived Value and Potential Contribution of Project Management Practices to Project Success. Project Management Journal, p. 37-49, 2006.

BERGGREN, C.; JÄRKVIK, J.; SÖDERLUND, J. Lagomizing. Organic Integration, and Systems Emergency Wards: Innovative Practices in Managing Complex Systems Development Projects. Project Management Journal, p. 111-122, 2008.

BOEHM, B. Get ready for agile methods, with care. IEEE Computer Magazine, Jannuary 2002, [S.I], p. 64-69, 2002.

BOEHM, B.; TURNER, R. Balancing Agility and Discipline: a guide for the perplexed. Boston: Addison-Wesley, 2004.

BOEHM, B.; TURNER, R. Management challenges to implementing agile process in traditional development organizations. IEEE Software, [S.I.], p. 30-39, set/out 2005

CARDEN, L.; EGAN, T. Does our literature support sectors newer to project management? The search for quality publications relevant to nontraditional industries. Project Management Journal, 2008.

CHICARINO, G. Z. O uso do Mind Map como ferramenta de gestão de projetos em áreas de consultoria e desenvolvimento de negócios e produtos, 2005.

CHIN, G. Agile Project Management: how to succeed in the face of changing project requirements. New York: Amacom, 2004.

CONFORTO, E. C.; AMARAL, D. C. Evaluating an agile method for planning and controlling innovative projects. Project Management Journal, v. 41, n. 2, p. 73-80, 2010.

CONFORTO, E. C.; AMARAL, D. C. Applying agile project management approach to NPD: the case of a small technology-based enterprise. In: International Product Development Management Conference - IPDMC, 16, 2009, Enschede, Netherlands. European Institute for Advanced Studies in Management. Proceedings... Netherlands: EIASM, 2009.

CONFORTO, E. C.; AMARAL, D. C.; SILVA, S. L. Roteiro Para Revisão Bibliográfica Sistemática: Aplicação no Desenvolvimento de Produtos e Gerenciamento de Projetos. In: $8^{\circ}$. Congresso Brasileiro de Gestão de Desenvolvimento de Produto CBGDP Porto Alegre, RS, Brasil, 2011. Instituto de Gestão de Desenvolvimento do Produto - IGDP. Anais... Porto Alegre: IGDP, 2011. 
COHN, M. Agile Estimating and Planning. New York: Prentice Hall PTR, 2005.

DAWSON, R.; DAWSON, C. Practical proposals for managing uncertainty and risk in project planning. International Journal of Project Management. v. 16, n. 5, p. 299310, 1998.

DECARLO, D. Extreme Project Management: using leadership, principles, and tools to deliver value in the face of volatility. San Francisco: Jossey Bass, 2004.

DVIR, D.; LECHLER, T. Plans are nothing, changing plans is everything: the impact of changes on project success. Research Policy. v. 33, n. 1, p. 1-15, 2004.

DVIR, D.; RAZ, T.; SHENHAR, A. An empirical analysis of the relationship between project planning and project success. International Journal of Project Management. v.21, n.2, p.89-95, 2003.

GANGULY, A.; NILCHIANI, R.; FARR, J.V. Evaluating agility in corporate enterprises. International Journal Production Economics, v.118, p.410-423, 2009.

GRIFFITHS, M. Teaching agile project management to the PMI. IEEE Computer Society. In: Agile Development Conference, 2005.

HASS, K. B. Managing Complex Projects - A New Model. Viena: Managements Concepts, 2009 (NAO TEM JOURNAL)

HIGHSMITH, J. Agile Project Management: creating innovative products. Boston: Addison-Wesley, 2004.

HODGETTS, P. Refactoring the development process: experiences with the incremental adoption of agile practices. IEEE Computer Society. In: Agile Development Conference, 2004.

HUTTENLOCHER, D.; SPOONHOWER, D. Principles and Practices of Software Development. Computer Science Department and Johnson Graduate School of Management. Cornell University. Ithaca, NY, 2002.

KIOPPENBORG, T.; OPFER, W. The current state of project management research: trends, interpretations and predictions. Project Management Journal, v. 33, n. 2, p. 5-18, 2002.

KOLLTVEIT, B.; KARLSEN, J.; GRONHAUG, K. Perspectives on project management. International Journal of Project Management, v. 25, n. 1, p. 3-9, 2007.

LEACH, L. Lean project management: eight principles for success. Idaho: Advanced Projects Boise, 2005.

MAFAKHERI, F.; NASIRI, F.; MOUSAVI, M. Project Agility assessment: an integrated decision analysis approach. Production \& Planning Control, v. 19, n. 6, p. 567-576, 2008. 
MAYLOR, $\mathrm{H}$. Beyond the Gantt chart: project management moving on. European Management Journal, v. 19, n. 1, p. 92-100, 2001.

MURCH, R. Project Management - Best Practices for it professionals. New York: Prentice Hall, 2001.

NERUR, S.; Mahapatra, R. Challenges of Migrating to Agile Methodologies. Communications of the ACM, v. 48, n. 5, p. 73-78, 2005.

OXFORD DICTIONARIES, Oxford University Press, 2010.

PERMINOVA, O.; GUSTAFSSON, M. \& WIKSTRÖM, K. Defining uncertainty in projects - a new perspective. International Journal of Project Management, v. 26, n. 1, p. 73-79, 2008.

PROJECT MANAGEMENT INSTITUTE - PMI. PMBoK Guide. A Guide to the Project Management Body of Knowledge. $4^{\text {th }}$ ed. Pennsylvania: Project Management Institute, 2008.

PROJECT MANAGEMENT INSTITUTE. Um Guia do Conhecimento em Gerenciamento de Projetos (Guia PMBOK®). Quarta Edição. Pennsylvania: 2008.

ROZENES, S.; VITNER, G.; SPRAGGETT, S. Project control: literature review. Project Management Journal, v. 37, n. 4, p. 5-14, 2006.

SALO, O.; Abrahamson, P. An iterative improvement process for agile software development. Software Process Improvement Practice, v. 12, p. 81-100, 2007.

SAUSER, B. J.; REILLY, R. R.; SHENHAR, A. J. Why projects fail? How contingency theory can provide new insights - a comparative analysis of NASA's Mars Climate Orbiter loss. International Journal of Project Management, v. 27, [s.n], p. 665-679, 2009.

SIBBET, D. Visual Meetings - How Graphics, sticky notes \& Idea mapping can transform group productivity. New Jersey: John Wiley \& Sons, 2010.

SHENHAR, A. Strategic project leadership: toward a strategic approach to project management. R\&D Management, v. 34, n. 5, p. 569-578, 2004.

SHENHAR, A.; DVIR, D. Reinventing Project Management: the diamond approach to successful growth and innovationt. Boston: Harvard Business School Press, 2007.

SMITH, P.G. Flexible Product Development - building agility for changing markets. San Francisco: Jossey-Basso, 2007.

STEFFENS, W.; MARTINSUO, M.; ARTTO, K. Change decisions in product development projects. International Journal of Project Management, v. 25, n. 7, p. 702-713, 2007. 
SUIKKI, R.; TROMSTEDT, R.; HAAPASALO, H. Project management competence development framework in turbulent business environment. Technovation, v. 26, n. 5, p. 723-738, 2006.

SCHWABER, K. Agile Project Management with SCRUM. Washington: Microsoft Press, 2004.

THOMKE, S.; REINERTSEN, D. Agile product development: managing development flexibility in uncertain environments. California Management Review, v. 41, n. 1, p. 830, 1998.

WILLIAMS, T. The need for new paradigms for complex projects. International Journal of Project Management, v. 17, n. 5, p. 269-273, 1999.

WINTER, M.; SMITH, C.; MORRIS, P.; CICMIL, S. Directions for future research in project management: the main findings of a UK government-funded research network. International Journal of Project Management, v. 24, n. 8, p. 638-649, 2006.

WordNet®, Princeton University, 2010.

WYSOCKI, R.; MCGARY, R. Effective Project Management: traditional, adaptive, extreme. Indianda: Wiley Publishing, 2007. 